\title{
Technology as the key to women's empowerment: a scoping review
}

\author{
April Mackey* ${ }^{*}$ and Pammla Petrucka
}

\begin{abstract}
Background: Information and communications technologies (ICTs) have empowered people to communicate and network at a global scale. However, there is lack of in-depth understanding of the use of ICTs for women's empowerment. This study examines how the concept empowerment is defined, utilized and measured in research studies, the existing evidence on the use of ICTs for women's empowerment and the gaps in knowledge at the global level.

Methods: The authors' conducted a scoping review using the Arksey and O'Malley methodology. The search identified papers from ten databases, including Scopus, Embase, ABI Inform, Soc Index, Sociological Abstracts, Gender Studies, Springer Link, PsychInfo, Science Direct, and Academic Search Complete over the period of 2012-2018. Search criteria included articles that focused on women's empowerment and utilized technologies as interventions. Out of a total of 4481 articles that were initially identified, 51 were included.
\end{abstract}

Results: Technology played a variety of roles in supporting the development of women's capacities and resources. Results revealed the use of ICT interventions in the overarching areas of outreach (e.g., health promotion), education (e.g., health literacy opportunities), lifestyle (e.g., peer coaching and planning), prevention (e.g., screening opportunities), health challenges (e.g., intimate partner violence apps), and perceptions of barriers (i.e., uptake, utilization and ubiquity to ICTs for women). Despite the positive use of technology to support women in their daily lives, there was a lack of consensus regarding the definition and use of the term empowerment. The concept of empowerment was also inconsistently and poorly measured in individual studies making it difficult to determine if it was achieved.

Conclusion: This scoping review provides a comprehensive review of current and emerging efforts to use ICTs to empower women. The findings suggest a need for collaborative efforts between researchers, program implementers and policy makers as well as the various communities of women to address the persistent gender disparities with respect to ICTs.

Keywords: Information and communications technology, Impact, Women empowerment, Scoping review

\section{Background}

The term women's empowerment emerged in the 1970s in response to the need for social justice and gender equality [1, 2]. As the term evolved in the 1990s, it was increasingly applied to women who were oppressed and lacking the freedom of choice and action to shape their lives, as well as to discuss women's participation across

*Correspondence: April.mackey@usask.ca

University of Saskatchewan, 100-4400 4th Ave, Regina, SK S4T 0H8,

Canada multiple sectors in society. More recently it has been used as an outcome and a goal to be achieved is to balance the scales of gender equality and equity. For this research, the definition used regarding women's empowerment is a process by which women who have experienced oppression acquire the ability to make autonomous and strategic life choices based on their personal priorities. Empowerment is achieved when a woman has the resources, agency, and capabilities to execute decisions on matters of importance $[3,4]$. 
Globally women are more likely to experience less favourable social determinants of health (such as overrepresentation of women in low-paying, insecure employment; lower education and literacy levels amongst rural and immigrant women) than their male counterparts. Women carry the bulk of responsibility for raising children and meeting household obligations, which, globally, contribute to this continued disadvantage [5-7]. Due to a lack of affordable and quality daycare, women are over-represented in part-time work force, and often remain within low-income bracket [5-8]. Gender, as a social determinant of health, is influenced by the "gendered" norms of the roles, personality traits, attitudes, relative power, and influence that society ascribes to it $[9,10]$. The transition from the Millennium Development Goals to the Sustainable Development Goals (SDGs) in 2015 saw the emergence of Target 5 which aims to "Achieve gender equality and empower all women and girls" (p. 20) [11]. A major SDG indicator supporting attainment of women's empowerment is "enhancing the use of enabling technology by increasing the proportion of women and girls who have access" (p. 20) [11].

Information and communication technologies (ICTs) have catalyzed communication and networking between and among people on a global scale. However, as ICTs have become ubiquitous and grown in both type and access, a digital divide has emerged. This divide parallels gaps in social contexts, such as income and education, as those who use and benefit from access to technologies often have other resources more readily available [12]. This divide widens the inequity and inequality gaps based on gender, age, disability, or socioeconomic status [13, 14].

Women's empowerment and ICTs have been the subject of global goals, discussions, and debates for many decades [15, 16]. Global discussions, such as the 1995 World Conference on Women: Beijing Declaration and Platform for Action, deliberated and advocated for the inclusion of women in the information society in order to fully achieve women's empowerment in connection with ICT. In 2013, 200 million more men had access to the internet than women [17]. Women use ICTs much less frequently and intensely than men [18-21] In 2016, the International Telecommunications Union (ITU) stated that the percentage of women gaining access to ICT is actually decreasing-with women utilizing ICTs $11 \%$ less than men in 2013 and 12\% less than men in 2016 [19]. The most recent 2018 report indicated that the overall proportion of internet usage for women was $12 \%$ lower than men [19].

The extant evidence lacks sufficient depth and detail as to exactly how ICTs are being used by women and why they use it less frequently. An important aspect of empowerment in the context of ICTs is gaining a clearer picture as to the type of technologies and technological interventions being used by women. Many authors agree that improved access to ICTs can assist in providing women with employment resources and opportunities that could narrow the gender wage gap, assist in making education and health information more accessible, contribute to the end of violence against women, and lead to women's empowerment and leadership [15, 22-24].

The objectives of this research were to: determine how the concept of empowerment is defined, utilized, and measured in research studies; explore existing evidence regarding the use of ICTs as interventions towards achieving women's empowerment; and explore the gaps in knowledge and research on this topic from an individual, community, and global perspective.

\section{Methods}

This research involved a scoping review, which is methodologically similar to a systematic review, to provide a rigourous synthesis of existing evidence $[25,26]$ For the purpose of this study, the scoping review framework used was described by Arksey and O'Malley [27] as a five-step process with an optional sixth step. These steps include: (1) identifying the research question, as the starting point to guide the search strategy; (2) identifying relevant studies, which involved the development of a comprehensive search strategy to ensure accurate and complete results; (3) selecting studies, which involved developing a-priori inclusion and exclusion criteria that were revised throughout the review process, as familiarity with the evidence increased; (4) charting the data, which involved charting and sorting key material from the results into themes and trends; (5) collating, summarizing, and reporting the results, which involved presenting the results as a narrative; and (6) consulting with relevant stakeholders, which is contingent upon time and resource considerations. For the purposes of this research, the sixth step was not performed.

\section{Review protocol, team, and management}

To ensure transparency, rigour, reproducibility, and consistency, protocols were developed prior to the start of the research, for the inclusion criteria, search strategy, and data characterization. This helped to ensure an unbiased approach to the search protocol and to enhance rigour [27]. These are available upon request. The scoping review was conducted by a team of individuals with multi-disciplinary capabilities in nursing, knowledge synthesis methodologies, and ICTs. The primary reviewers included the lead and co-authors, as well as one research assistant. In addition, a University librarian was consulted throughout the search term selection process 
to ensure completeness and accuracy of search terms as well as a comprehensive and complete search strategy.

Any and all potentially relevant citations identified throughout all stages were imported into EndNote ${ }^{\mathrm{TM}}$, a reference management software, where duplicates were removed by the program and then double checked, and manually removed by the lead author; the list of citations was then imported into a web-based electronic systematic review management platform, DistillerSR ${ }^{\mathrm{TM}}$. The screening for article relevance, up to the data extraction stages, were conducted using this software. Two reviewers (i.e., lead author and research assistant) were involved throughout the selection and analysis process to ensure consistency, adherence to the inclusion/exclusion criteria, relevance to the research question, as well as the categorization of data into themes and patterns. As part of this process, all articles were screened by the lead author and research assistant. Any discrepancies were brought forward to the co-author who made an independent decision whether to include or exclude the article.

\section{Review intent and scope}

This was part of a broader study aimed at addressing the following question: What is the global impact of ICTs on women's empowerment? The current review aimed to examine the concept of empowerment, while exploring the evidence on ICTs as interventions for achieving women's empowerment at the individual, community, and global levels.

\section{Search strategy}

The authors ensured identification of relevant and suitable publications by creating a search strategy protocol prior to retrieving evidence from a variety of sources. As per Arksey and O'Malley [27], the following avenues were reviewed as part of the search strategy: searching relevant electronic databases, reviewing reference lists of pertinent articles to identify additional sources, and manually searching key journals.

To ensure the search was comprehensive, the following databases, available through the University of Saskatchewan library, were searched on November 30, 2016 and updated on January 1, 2018: Scopus, Embase, ABI Inform, Soc Index, Sociological Abstracts, Gender Studies, Springer Link, PsychInfo, Science Direct, and Academic Search Complete. The COCHRANE Library was also searched for any relevant trials in the trial registry. Limits placed on the search included: English only, no book reviews, publications dated 2012-2017, and the protocol was pretested in Scopus and Soc Index using select key words including "women" and "empowerment" and "technology". An illustration of the search term strategy is presented in Table 1.
Search terms were drawn from the research question, as well as from lengthy discussions with the university librarian and expanded upon based on a cursory search of two databases. To determine the range and breadth of key terms, an initial limited search of two databases was conducted yielding several papers. These databases were determined in consultation with the university librarian and included Scopus and Gender Studies. These papers were then analyzed for similar keywords, definitions, analogies, and index terms that were relevant synonyms to the initial search words [28, 29]. These additional terms were added to a master list that informed the final search strategy. Specifically, for the term empowerment, keywords were chosen that could provide results that included a lack of empowerment as well, thus the inclusion of "barrier" and "disempower". The other search terms came directly from key articles and databases and were demonstrated to be the most common variations on the term "empower". An additional term that was used interchangeably with "empower" was "agency", however, as this term is used more frequently in conjunction with organizations and not empowerment, it was removed from the search term list.

The ability of the electronic database search to identify all relevant primary research was verified by hand searching the reference lists of eight key peer reviewed articles and nine key electronic journals that were flagged through the initial test search as well as the main search. The journals were chosen based on their relevance to the research question as well as their scholarly nature. The initial three identified journals were: Community Informatics, Gender and Development, and Journal of Women in Culture and Society. Subsequent journals were identified and selected for a hand-search once the initial search was completed. These were: Gender, Technology \& Development, Computers in Human Behaviour, American Journal of Health Behaviour, American Journal of Public Health, and Women's Health Issues. These journals were then reviewed for additional articles potentially not identified through the database search; this included entering the general search into journal databases.

Additional grey literature was identified by handsearching the websites of the Association for Computing Machinery Digital Library Journals and Conference Proceedings, the UN Women, Status of Women Canada, the United Nations Development Program, the International Center for the Research of Women, the Girls Action Foundation, the Information and Communications Technology Council, the ITU, and the International Development Research Center for primary research reports, guidelines, situation reports, and referenced publications that were not already included. 
Table 1 Search Term Strategy

\begin{tabular}{|c|c|c|c|c|}
\hline Women Search Terms & & ICT Search Terms & & Empowerment Search Terms \\
\hline${ }^{*}$ searched with OR & "AND" & ${ }^{*}$ searched with OR & "AND" & ${ }^{*}$ searched with OR \\
\hline Wom?n & & Technolog* & & Empower* \\
\hline Female* & & Information technolog* & & Disempower* \\
\hline Girl* & & $\begin{array}{l}\text { "information communications } \\
\text { technolog*" }\end{array}$ & & Barrier* \\
\hline Maternal & & "ICTs" & & Enable* \\
\hline & & "social media" & & Self concept \\
\hline & & mobile & & Self efficacy \\
\hline & & handheld & & Capacit* \\
\hline & & telehealth & & Emancipat* \\
\hline & & computer & & \\
\hline & & Smartphone & & \\
\hline & & Digital & & \\
\hline & & Internet & & \\
\hline & & Telecommunication* & & \\
\hline & & "world wide web" & & \\
\hline & & Laptop & & \\
\hline & & ICT4D & & \\
\hline & & "web-based" & & \\
\hline & & Iphone & & \\
\hline & & Ipad & & \\
\hline
\end{tabular}

*At end of word = truncation, any number of letters (e.g. capacit* will find capacity or capacities); ? at end of beginning of word is used to represent one or more other characters in a search term (e.g. wom?n will find women or woman)

Limits included: 2012-2017, English language, no books/book reviews

\section{Study selection: relevance screening and inclusion criteria}

The focus of the study selection was locating published and unpublished academic articles, which may have any type of study design, including qualitative, quantitative, or mixed methods. The initial pool of results included a total of 4481 citations. An initial set of inclusion and exclusion criteria were developed a-priori to screen abstracts and titles of citations which were refined during each review of the pool of articles. Research articles were initially considered relevant if they included women's empowerment and/or information and communication technology concepts in the title or abstract of the publication. Synonyms for these concepts were created in consultation with the librarian to ensure a robust search strategy for maximum location and inclusion of studies. Given the evolving nature of ICTs and their role in interventions, the authors wanted the articles to reflect a recent knowledge base, therefore the timeframe of 20122016 was chosen, which was later expanded to December 31, 2017 as the review progressed. The results were also filtered to include English only content.

\section{First screen: inclusion criteria}

The inclusion criteria created for the first level of study selection were driven by the review topics, specifically, women, empowerment, and ICTs. According to the Joanna Briggs Institute (JBI) (2015), the inclusion criteria should be based on three themes, also known by the acronym of PCC: (a) participant description, (b) concept, which is likened to the phenomena of interest, and (c) context. The inclusion criteria used in the first level of selection were country of publication, date of publication (2012-2017), and the use of both of the following concepts in the title or abstract of the publication: women's empowerment and/or information and communication technology. At this stage, the lead author looked for the presence of the key words in the title and/or abstract. The use of these keywords as inclusion criteria was designed to be intentionally broad to provide a sense of what publications linked the two concepts (i.e., women's empowerment and ICTs).

\section{First screen: study selection}

On first review, the initial pool of articles was subjected to a staged process to ensure studies were selected that were relevant to the research question and met the inclusion criteria. Articles were first excluded based on duplication within the initial search results. This exclusion was conducted using the search tools feature within the electronic database, but also within the reference 
management program Endnote ${ }^{\mathrm{TM}}$ and then manually by the lead author. The inclusion criteria were applied to the title and abstract of the publication. Any title or abstract that did not meet the inclusion criteria was removed from further review and consideration. All articles excluded by the criteria were sent to the research assistant who confirmed the exclusion. Any disagreements or contradictions between the primary author and the research assistant were thoroughly discussed, with both parties having to agree to the inclusion before the publication could be added back into the pool of articles to move on to the next stage. Additionally, if an article could not be excluded based solely on the title or the abstract, the full article was reviewed for relevance to the research question and inclusion criteria. These latter two points did not prove to be an issue as there were no disagreements.

\section{Second screen}

The remaining pool of articles was then reviewed a second time by applying a second level of inclusion criteria to the title as well as the abstract. It is common and encouraged as part of the scoping review process to generate increased cumulative familiarity with how concepts are presented within the evidence. This, in turn, informed the decisions that were made regarding the inclusion or exclusion criteria in the subsequent stage. Much of the articles after the first level of elimination included technology as a passive aspect of the study and not one that women actively participated in. It was important for the authors that the technological aspect of each study be an intervention that women could engage in towards building self-efficacy and capacity. This informs current gaps within the evidence that speak to how women are using technologies to support their empowerment. As such, this set of inclusion criteria focused on technology as an intervention and women as active participants in the study instead of just the word "women" found throughout the first set of criteria.

\section{Final screen}

For the final review of the full text articles, based on the content and findings in the scoping review process, an additional criterion was included. The authors wanted to explore how the social determinants of health informed and supported the concepts of women, empowerment, and ICTs. At this stage, it was noted which social determinants of health, if any, were present in each article. The list of social determinants based on the Government of Canada (2019) criterion was utilized as a reference for this portion of the process, such as employment and working conditions; income and social status; social supports and coping skills. The remaining 59 articles all had social determinants of health. A subsequent review resulted in 14 of the 59 articles being eliminated from consideration as they did not meet the inclusion/exclusion criteria. Rather than focus on a range of these determinants, the authors decided to include all 45 articles and to then review the implications of this finding in the analysis (Fig. 1).

\section{Re-run searches}

Due to the extended time to conduct the review, the authors included re-run searches for each database up to January 1, 2018. A total of 573 articles were found in all 10 of the main electronic databases. Using the inclusion and exclusion criteria previously described all but six articles were eliminated through the first and second stages in the review process. The final total number of articles included in the analysis was 51 .

\section{Study characteristics, extraction, and charting}

The final step in the Arksey and O'Malley's [27] scoping review framework was to collate and summarize the results for presentation and discussion. Each selected article was summarized in a customized data characterization utility form to guide data extraction. The goal of this step was to determine and chart factors to be extracted from each article to help answer the research question $[26,27,30]$. The charting of data was an iterative and exploratory process in which the data charts were continuously updated to ensure completeness and accuracy $[26,30]$. Data extracted from the charts included year of publication, country of study, implications for policy and practice, types of ICT interventions, demographics, empowerment (definition, as a design consideration, and measures), and social determinants of health (presence and description within in the study). All data were then analyzed using thematic analysis and the main ideas refined over several iterations. The data were then mapped using tabular and visual presentations of the main conceptual categories followed by a narrative summary describing how the results related to the research question and objectives.

\section{Results}

\section{Demographics and study characteristics}

The geographic range of the included evidence was global; however, $41.1 \%(21 / 51)$ described research conducted in the USA. Seven studies were conducted in India, three in Australia, three in Sweden, and two in Canada. One study was conducted in each of the following countries: Finland, Ghana, Italy, Japan, Nepal, Netherlands, Nigeria, Singapore, South Korea, Sri Lanka, Tanzania, Thailand, Uganda, and United Kingdom.

Collation and comparison of demographics was difficult due to a lack of consistency in reporting. However, 
Database searches

Total: 4481

ABIInform $=219$

Academic Search Complete $=1157$

Embase $=1239$

Gender Studies $=132$

Psychinfo $=666$

Science Direct $=21$

Scopus $=307$

Sociological Index $=183$

Soc. Abstracts $=147$

SpringerLink $=130$

Handsearch Journals $=202$

Reference lists from key articles $=78$

Citations Screened $=3741$

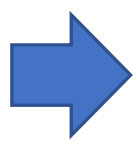

Citations Screened=1103

Citations Screened $=305$

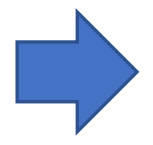

Citations Screened $=57$

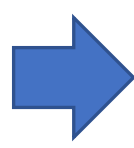

Relevant Articles, peer reviewed $=45$

*ReRun Search $=573$

Citations excluded due to duplicates and inclusion/exclusion $=$ 567
Duplicates removed $=1658$

\section{Citations Excluded}

$590 \mathrm{~d} / \mathrm{t}$ wrong date imported into Endnote accidentally

$2036+12$ grey lit $=$ not relevant to topic
Citations Excluded

$797=$ not relevant to topic $\&$ did not meet criteria

$1=$ error citation within database

Citations Excluded

$246=$ Did not meet criteria

$2=$ no full text available

Citations Excluded

$$
=12
$$

Theoretically based $=5$

No technology intervention $=2$

No concept of empowerment present $=3$

Not primary research $=1$

Not specific to women $=1$

$$
\begin{aligned}
& \text { Total Articles } \\
& \text { Included in } \\
& \text { Analysis }=51
\end{aligned}
$$

Fig. 1 PRISMA Flow for Screening Process 
all articles described the demographics of women who were the primary focus of the study. Seventy eight percent of the articles (40/51) reported on some or all of the descriptive study characteristics. The age of participants was frequently reported although there were inconsistent age groupings across the studies. Some articles only reported the mean age of participants, while others provided only an age range. The lowest mean age reported was 24 years while the highest was 59.6 years; the categories ranged from less than 16 years to 64 years and older. It is difficult to compare these age ranges as the categories varied in the included articles, and it was unclear as to whether age was simply a descriptive statistic designed to describe the sample or whether it was reflected upon consistently in terms of the overall implications to the study.

Missing from the studies were the perspectives and participation of women who could not access, afford, and/or purchase an ICT device as well as effectively and fully utilize it to support their empowerment. Exclusion criteria used in the individual 51 studies illustrate that women not already owning a mobile device, computer, or tablet were eliminated from participating in the research.

\section{Empowerment definition}

In the included studies, the concept of empowerment was used incongruously with terms like self-concept, self-esteem, and self-worth, sometimes by the same author in the same study, which further limited our ability to achieve a uniform definition for the purposes of this research. Less than one quarter (12/51) of the studies used the term "empower(ment)" in their definition of the concept of interest. These studies defined empowerment as a process but with different foci: as individuals having choice or control over their decisions [31-38], as being multi-dimensional and influencing a variety of areas [34, $37,39,40]$ or with a focus on building individuals' capacities, including internal and external resources [39-42].

The remaining studies described empowerment in a more indirect way, never including the term "empower" or "disempower." Instead, the term empowerment was described in synonymous terms, for instance, half $(28 / 51)$ described the concept of empowerment as the process of enabling a sense of self-efficacy or self-worth in the ability to overcome barriers to resources, as well as the barriers to decision-making control [43-70]. One fifth (11/51) described empowerment as the process enabling a sense of self-efficacy or self-worth in the ability to overcome barriers to control over resources [71-81].

\section{Measures of empowerment}

All studies considered the concept of empowerment in their design; $80 \%(41 / 51)$ of the articles considered empowerment as a primary outcome of the study. No measures of empowerment were specifically cited in any of the articles, beyond the measures of the behaviour being studied. Several studies included various measures of self-efficacy (i.e., childbirth [74], physical activity [56, 67, 71, 74, 80, 81], intimate partner violence [72], caregiving [75], barrier [55, 56], health [78], and chronic disease management [77]). The authors of the articles did not compare the different types of self-efficacy scales for validity of empowerment. The diversity of the scales illustrates a focus on improving efficacy of individual behaviors rather than the holistic empowerment of women.

\section{ICT interventions to support women's capacity and tools}

The articles described a range of supportive ICT interventions, though with inconsistent and overlapping classification. The specific types of interventions covered in the 51 articles included web-based devices (17), the internet (19), particular websites (3), blogs (1), text messaging (4), telemedicine (1), video (1), apps (5), social media (2), computers (6), email (1) and Fitbit ${ }^{\mathrm{TM}}(1)$. Our categorization of ICTs focused on how the specific interventions were utilized in the day to day lives of women and were obtained from a thematic analysis of the types of ICTs used by women in the studies. The themes included (1) Outreach; (2) Education; (3) Lifestyle (4) Health Challenges; (5) Prevention; and (6) Perception of Barriers.

\section{Outreach}

Ten of the 51 articles reviewed described supportive ICT interventions as a means of outreach or connecting with clients in the community. Common themes in this section included supporting women where they are at in the community, in terms of their social position, to enhance positive health behaviours with technological assistance, as well as overall enhanced accessibility to ICTs. This was accomplished through Cognitive Behavioural Therapy using computers [46], and web-based decision aid for understanding fetal anomalies [47]. Educational text messages were sent to encourage breastfeeding [79], and general health promotion interventions were delivered as well $[43,44,56,65,73,75,80]$.

\section{Education}

Six articles described supportive ICT interventions that delivered various health information, through smartphones or other web-based devices. These included Facebook $^{\mathrm{TM}}$ virtual learning systems [34], psychoeducation for breast cancer patients [35], as well as interactive voice response as a tool for improving access to healthcare in remote areas [59]. Other interventions included English language programs [70], antenatal perineal massage 
support groups [76], as well as support for enhancing doctor-patient relationships. [64].

\section{Lifestyle}

Twelve articles described supportive ICT interventions that focused on behavioural outcomes related to general lifestyle areas, using web-based devices. Commonly, the interventions provided some form of external support for women to improve their overall way of being healthy. These included improving nutrition knowledge and behaviours $[67,69]$, promoting healthy food planning, shopping, and eating behaviours [54], interventions for weight loss behaviours, [45] and engagement with physical activity coaching [55, 71, 74]. Many of the interventions focused on social networks $[9,48]$, for example, peer support for building social capital [52], and promoting social behaviours through an iPad book club [81].

\section{Health challenges}

Eleven articles described ICT interventions that focused on using web-based devices to address specific health challenges. The health challenges largely focusing on ways to enhance maintenance of women's health, for example, self-paced education programs for those who experience intimate partner, as well as dating violence $[33,41,72]$, and educational training to enhance understanding and management of chronic illness [77]. The interventions addressing health challenges were concentrated on those that affect women only, for example educational training for patients with breast cancer [38, 61], health modules for those with breast cancer [78] and stress incontinence [63] and advanced care planning for women with ovarian cancer [49].

\section{Prevention}

A few articles (3/51) described ICT interventions that focused on preventing specific health challenges using web-based devices. One intervention focused on the prevention of sexual and reproductive illness using education information [57]. Another encouraged vaccination behaviors and immunization with educational information [58] as well as the prevention of pre-eclampsia in rural developing countries using diagnostic tools [51]. One study focused on utilizing mobile phones to manage money transfers to support transport of women with fistula to urban hospitals [60] and another examined electronic health records to improve breast cancer screening [53].

\section{Perceptions of barriers}

Nine articles described ICT interventions that focused on the perception of barriers to ICTs that assist women in advancing their understanding and use of ICTs. These studies focused on the perceived barriers and understanding of the role of mobile phones, $[42,66]$ the awareness of gender-based barriers in telemedicine [68], the development of women through mobile phones [32, $40]$, as well as the connection with women in the community apps [50].

\section{Discussion}

\section{Concept of empowerment}

Empowerment is a multi-dimensional and contextual concept that is internal by nature, varies in meaning, and reflects how women self-ascribe it to themselves. From the outset of the review, search terms had to include words beyond simply "empower[ment]" as much of the initial searching revealed synonyms including selfefficacy, self-worth, self-concept, and/or capacity. This inconsistency in the use of the term empowerment yields a lack of consensus on how empowerment is understood which impacts how research studies and interventions are structured and delivered to ensure maximum effectiveness and generalizability. While none of the studies included in the review indicated the broader negative outcomes related to the use of ICT, the literature supports a flip side to using technology to empower women. For example, technological advances are disproportionately accompanied by female-directed cyber abuse [82, 83].

Evidence that women of poor socio-economic status are being left out of research studies and programs that aim to support women's empowerment, highlights that targeted access and funding for at risk populations (such as sub-populations of women) are essential considerations in policy and program development across individual, community, and global contexts. This also reflects biases in terms of the population sub-groups in research studies that aim to advance empowerment. Opportunities exist for further evaluation of how empowerment is being measured and used in conjunction with ICTs, as well as which frameworks are being used to guide research in this area. The lack of specific measures of empowerment reflects a barrier, not only regarding how strategies for empowerment are understood and implemented, but how researchers know whether empowerment has been achieved. The finding underscores a need for a standardized tool for measuring the level of women's empowerment.

\section{ICTs to improve empowerment}

Empowerment through ICTs has the potential to cross multiple sectors, both private and public. The complexity of empowerment and ICTs, as they relate to the root issues of inequities, suggests the need for collaborative, 
multi-sectoral involvement. These partnerships consider the contextual factors that act as facilitators and barriers for women in all types of communities. Interagency partnerships are uniquely suited to develop interventions aimed at enabling women to make better use of ICTs. These interventions should include information on access to education, facilities for education regarding entrepreneurship, employment opportunities, and health and other government health resources. Governments partnering with private telecommunication agencies through subsidization could provide discounted or refurbished devices for women who are deemed disadvantaged. Funding may also benefit those who experience difficulty in obtaining mobile devices as well as in accessing interventions aimed at enhancing the use of ICT. For example, funding is needed to support the cost of accessing services, low-cost devices, or the provision of Subscriber Identity Module (SIM) cards. Alternatively, governments should support and encourage private mobile operators through tax exemptions and other benefits to facilitate better mobile services and infrastructure in rural, remote, and urban areas. Providing accessible computer sites within communities or in schools is another way to bridge the gap in access to and use of ICT. These strategies not only help in improving the overall status of girls and women but also influence overall empowerment and development of the community.

Though ICT is not the only factor that can support women's empowerment through capacity building, women who do not have access to or who cannot afford ICTs, are potentially disempowered due to a lack of voice and participation within the information sphere. Exclusion of such women from research limits the measurement of the true impact of ICTs on empowerment and generalizability of findings. Continued research regarding empowerment involving more advantaged sub-groups of women does not address the inherent issues of oppression of women within society and further disempowers those under-represented groups. Local policies (such as affordable internet as a basic need; basic digital literacy education embedded in local curricula) have the greatest potential of improving the uptake of ICTs, as this process occurs initially at the individual level.

\section{Individual, community, and global knowledge}

Local and national governments need to invest in information gathering tools that inquire how and why women are using technology to support their lives and families. Equally important is the inquiry of women's perceptions regarding how they prefer to use ICTs to improve their lives or the barriers they experience in the process. A global survey undertaken by the UN Statistics Division in 2011 indicated that only 30 percent of countries regularly produce sex-disaggregated statistics (such as male:female access to ICT; digital literacy by gender) and existing data collection approaches do not incorporate qualitative components that highlight the voices of women [84].

Future data should be translated into gender sensitive policies that support equal access and use of ICTs. The development and implementation of such policies should involve representation of women from all socioeconomic backgrounds and ages to ensure maximum impact. Examples include policies that allow women to effectively access and participate in ICTs within society, the delivery of ICTs at a reasonable cost for all, as well as policies that regulate the cost and provision of services linked to ICTs such as availability of cell phone, easily accessible WiFi sites, and cost-effective internet plans.

\section{Limitations}

While scoping reviews examine the breadth of evidence available on a topic, they do not factor in the depth or quality of that evidence [25-27, 30]. Some authors have argued that scoping reviews should include an assessment of quality; however, Armstrong et al. [25] contend that this decision should depend on the resources available for the review as well as the purpose of the scoping review itself. The quantity of data that is generated in a scoping review can be significant and so it is important to find a balance between providing an overview of all types of evidence found and providing detailed data and assessment of a smaller number of studies [25]. Scoping studies also lack a thorough evaluation of the quality of results, instead producing a narrative account of all available evidence [26, 27]. This approach serves to ensure that all resulting evidence is included in the review and does not limit the end number of articles, as in a systematic review.

\section{Conclusions}

The diversity of technological interventions utilized to support empowerment is infinite and there is no limit to how ICTs can be implemented in daily lives. This study is novel and essential as it comprehensively describes efforts to use ICTs to empower women, and the imperative for collaborations between researchers, program implementers and policy makers to address the persistent gender disparities in the access to and use of technologies. This research provides a foundation for future research on the concept of empowerment with ICTs in critical areas of outreach, education, lifestyle, health challenge, prevention, and perception of barriers. Outreach was linked to positive health behaviours such as health promotion and decision-making applications. Education interventions varied from learning systems to health relationships for knowledge sharing. Lifestyle ICT interventions 
were related to external supports, often peer based, for improving healthful choices such as coaching and planning tools. Health challenges and prevention were relevant to specific challenges (e.g., intimate partner violence; chronic diseases) and health literacy issues (e.g., vaccine awareness; screening programs), respectively. The final theme of perceptions of barriers reflected experiences by participants respecting uptake, utilization, and ubiquity of ICTs. Each of these areas is well situated for future intervention research and each area brings focal points and imperatives to this emerging research agenda.

\section{Abbreviations}

SDG: Sustainable Development Goals; ICT: Information and communication technology; ICTs: Information and communications technologies; WHO: World Health Organization; UN: United Nations; ITU: International Telecommunication Union; GPS: Global Positioning System; SIM: Subscriber Identity Module.

\section{Acknowledgements}

Not applicable.

\section{Authors' contributions}

AM was involved in the conceptualization, analysis, and writing of the first draft of the manuscript, as well as all edits. PP assisted in analyzing and interpreting the data and was a major contributor in writing the manuscript. All authors read and approved the final manuscript.

\section{Funding}

Not applicable.

\section{Availability of data and materials}

The databases used in the study were all open access and included Scopus, Embase, ABI Inform, Soc Index, Sociological Abstracts, Gender Studies, Springer Link, PsychInfo, Science Direct, and Academic Search Complete. The datasets used and/or analysed during the current study are available from the corresponding author on reasonable request.

\section{Ethics approval and consent to participate}

Approval from the University of Saskatchewan Behavioural Research Ethics Board was waived for this study as the information retrieved was publicly available.

\section{Consent for publication}

Not applicable.

\section{Competing interests}

The authors declare that they have no competing interests.

Received: 4 December 2019 Accepted: 16 February 2021

Published online: 23 February 2021

\section{References}

1. Freire, P. (1970). Pedagogy of the Oppressed. (M. B. Ramos, trans.). New York: Continuum.

2. Grabe S. An empirical examination of women's empowerment and transformative change in the context of international development. Am J Community Psychol. 2011:49:233-45.

3. Kabeer N. Resources, agency, achievements: reflections on the measurement of women's empowerment. Dev Change. 1999;30(43):435-64

4. Mosedale S. Assessing women's empowerment: towards a conceptual framework. J Int Dev. 2005;17:243-57.

5. Lips $\mathrm{H}$. The gender pay gap: Challenging the rationalizations. Perceived equity, discrimination, and the limits of human capital models. Feminist Forum, 2013;68:169-185. https://doi.org/10.1007/s11199-012-0165-z
6. Marmot M, Allen J, Bell R, Bloomer E, Goldblatt P. WHO European review of social determinants of health and the health divide. The Lancet. 2012;380(9846):1011-29. https://doi.org/10.1016/S0140-6736(12)61228 $-8$.

7. Raphael D. (2012). Tackling Health Inequalities: Lessons from International Experiences. Toronto: Canadian Scholars' Press; 2012.

8. Statistics Canada. Economic well-being. 2015a. http://www.statcan.gc.ca/ pub/89-503-x/2010001/article/11388-eng.htm\#a4

9. Mikkonen J, Raphael D. Social determinants of health: Canadian facts. 2010. http://www.nsgamingfoundation.org/articlesReports/Report_Socia I_Determinants_of_Health_The_Canadian_Facts.pdf

10. Public Health Agency of Canada. What determines health? In: Population Health. http://www.phac-aspc.gc.ca/ph-sp/determinants/index-eng.php

11. United Nations. Sustainable Development Goals Report. https://susta inabledevelopment.un.org/content/documents/21252030\%20Age nda\%20for\%20Sustainable\%20Development\%20web.pdf

12. Dixon LJ, Coorea T, Straubhaar J, Covarrubias L, Graber D, Spence J, Rojas V. Gendered spaces: the digital divide between male and female users in internet public access sites. J Comput Med Commun. 2014;19(4):991-1009.

13. Fang ML, Canham SL, Battersby L, Sixsmith J, Wada M, Sixsmith A. Exploring privilege in the digital divide: Implications for theory, policy, and practice. The Gerontologist. 2018;59(1):e1-15. https://doi.org/10.1093/ geront/gny037.

14. Pagán FJB, Martínez JL, Máiquez MCC. Internet use by secondary school students: a digital divide in sustainable societies? Sustainability. 2018;10(10):1-14. https://doi.org/10.3390/su10103703.

15. Brimacombe T, Skuse A. Gender, ICTs, and indicators: Measuring inequality and change. Gender Technol Dev. 2013;17(2):131-57.

16. Gurumurthy, A. (2004). Gender and ICTs: Overview report. Retrieved from http://www.bridge.ids.ac.uk/sites/bridge.ids.ac.uk/files/reports/CEP-ICTsOR.pdf

17. Intel. (2013). Women and web. Retrieved from http://www.intel.com/ content/dam/www/public/us/ en/documents/pdf/women-and-theweb.pdf

18. Hilbert M. Digital gender divide or technologically empowered women in developing countries? A typical case of lies, damned lies, and statistics. Women's Stud Int Forum. 2011;34(6):479-89. https://doi.org/10.1016/j. wsif.2011.07.001.

19. International Telecommunication Union. ICT facts and figures: 2017. 2017 https://www.itu.int/en/ITU-D/Statistics/pages/facts/default.aspx

20. Ono H, Zavodny M. Gender and the internet. Soc Sci Quart. 2009;84(1):111-21.

21. Wasserman IM, Richmond-Abbott MR. Gender and the internet: Causes of variation in access, level, and scope of use. Soc Sci Quart. 2005;86(1):252-70.

22. Gill, K., Brooks, K., McDougall, J., Patel, P., \& Kes, A. (2010). Bridging the gender divide: How technology can advance women economically. Retrieved from http://www.icrw.org/files/publications/Bridging-theGender-Divide-How-Technology-can-Advance-Women-Economically.pdf

23. Gurumurthy, A. (2006). Promoting gender equality? Some developmentrelated uses of ICTs by women. Development in Practice, 16(6).

24. Weiss $M$, Tarchinskaya $E$. The role of information technologies in changing the status of women to improve human conditions. In: Brocke JV, Stein A, Hofmann S, Tumbas S, editors. Grand societal challenges in information systems research and education. New York: Springer; 2015. p. 51-60.

25. Armstrong R, Hall BJ, Doyle J, Waters E. Scoping the scope of a Cochrane review. J Public Health. 2011;33(1):147-50.

26. The Joanna Briggs Institute. Methodology for JBI scoping reviews. 2015 http://joannabriggs.org/assets/docs/sumari/Reviewers-Manual_Metho dology-for-JBI-Scoping-Reviews_2015_v2.pdf

27. Arksey H, O'Malley L. Scoping studies: towards a methodological framework. Int J Soc Res Methodol. 2005;8(1):19-32. https://doi. org/10.1080/1364557032000119616.

28. Grimshaw J. A guide to knowledge synthesis. Canadian Institutes of Health Research. 2010. http://www.cihr-irsc.gc.ca/e/41382.html

29. Landa AH, Szabo I, Le Brun L, Owen I, Fletcher G, \& Hill M. An evidencebased approach to scoping reviews. The Electronic Journal of Information Systems Evaluation. 2011;10:4: 173-175. http://martinhill.me.uk/pubs/ EvidenceBasedApproachToLitReview.pdf 
30. Levac D, Colquhoun H, O'Brien KK. Scoping studies: Advancing the methodology. Implementation Science. 2010;5:1:1-9.

31. Chew HE, Ilavarasan VP, Levy MR. Mattering matters: agency, empowerment, and mobile phone use by female microentrepreneurs. Inf Technol Dev. 2015;21(4):523-42.

32. Handapangoda WS, \& Sisira Kumara A. From silence to voice: Examining the empowerment potential of mobile phones to women in Sri Lanka: The case of dependent housewives. 2012. https://mpra.ub.uni-muenc hen.de/41768/

33. Lindsay M, Messing JT, Thaller J, Baldwin A, Clough A, Bloom T, ... \& Glass N. Survivor feedback on a safety decision aid smartphone application for college-age women in abusive relationships. Journal of Technology in Human Services. 2013;31:4:368-388.

34. Vivakaran MV, Maraimalai N. Feminist pedagogy and social media: a study on their integration and effectiveness in training budding women entrepreneurs. Gend Educ. 2017;29(7):869-89.

35. Admiraal JM, van der Velden AW, Geerling J, Burgerhof JG, Bouma G, Walenkamp AM, ... \& Reyners AK. Web-based tailored psychoeducation for breast cancer patients at the onset of the survivorship phase: A multicenter randomized controlled trial. Journal of Pain and Symptom Management. 2017;54:4:466-75.

36. Nord JH, Riggio MT, Paliszkiewicz J. Social and economic development through information and communications technologies: Italy. J Comput Inf Syst. 2017;57(3):278-85.

37. Sarkar S. Beyond the "digital divide": the "computer girls" of Seelampur. Feminist Media Stud. 2016;16(6):968-83.

38. Siekkinen M, Kesänen J, Vahlberg T, Pyrhönen S, Leino-Kilpi H. Randomized, controlled trial of the effect of e-feedback on knowledge about radiotherapy of breast cancer patients in Finland. Nursing Health Sci. 2015;17(1):97-104.

39. Holbrey S, Coulson NS. A qualitative investigation of the impact of peer to peer online support for women living with polycystic ovary syndrome. BMC Women's Health. 2013;13(1):51.

40. Islam MK, \& Slack F. Women in rural Bangladesh: Empowered by access to mobile phones. In Proceedings of the 9th International Conference on Theory and Practice of Electronic Governance. 2016;March:75-84.

41. Choo E, Guthrie KM, Mello M, Wetle TF, Ranney M, Tapé C, Zlotnick C."I need to hear from women who have 'been there"': Developing a womanfocused intervention for drug use and partner violence in the emergency department. Partner Abuse. 2016;7(2):193.

42. Mehta BS, Mehta N. ICT and Socio-economic empowerment of rural women: case of mobile phone in India. Knowl Horizons-Econ. 2014;6(4):103-12.

43. Brown S, Hudson DB, Campbell-Grossman C, \& Yates BC. Health promotion text blasts for minority adolescent mothers. MCN: The American Journal of Maternal/Child Nursing. 2014;39:6:357-362.

44. Gold KJ, Normandin MM, Boggs ME. Are participants in face-to-face and internet support groups the same? Comparison of demographics and depression levels among women bereaved by stillbirth. Arch Women's Mental Health. 2016;19(6):1073-8.

45. Hearn L, Miller M, Lester L. Reaching perinatal women online: The healthy you, healthy baby website and app. J Obesity. 2014;2014:1-9.

46. Kim DR, Hantsoo L, Thase ME, Sammel M, Epperson CN. Computerassisted cognitive behavioral therapy for pregnant women with major depressive disorder. J Women's Health. 2014;23(10):842-8.

47. Åhman A, Sarkadi A, Lindgren P, Rubertsson C. "It made you think twice"an interview study of women's perception of a web-based decision aid concerning screening and diagnostic testing for fetal anomalies. BMC Pregn Childbirth. 2016;16(1):267.

48. Chib A, Malik S, Aricat RG, Kadir SZ. Migrant mothering and mobile phones: Negotiations of transnational identity. Mobile Media Commun. 2014;2(1):73-93.

49. Vogel RI, Petzel SV, Cragg J, McClellan M, Chan D, Dickson E, Geller MA. Development and pilot of an advance care planning website for women with ovarian cancer: a randomized controlled trial. Gynecol Oncol. 2013;131(2):430-6.

50. Ayiasi RM, Kolsteren P, Batwala V, Criel B, \& Orach CG. Effect of village health team home visits and mobile phone consultations on maternal and newborn care practices in Masindi and Kiryandongo, Uganda: A community-intervention trial. PloS One.
51. Jonas SM, Deserno TM, Buhimschi CS, Makin J, Choma MA, Buhimschi IA. Smartphone-based diagnostic for preeclampsia: a mHealth solution for administering the Congo Red Dot (CRD) test in settings with limited resources. J Am Med Inform Assoc. 2015;23(1):166-73.

52. Wollersheim D, Koh L, Walker R, Liamputtong P. Constant connections: piloting a mobile phone-based peer support program for Nuer (southern Sudanese) women. Aust J Primary Health. 2013;19(1):7-13.

53. Atlas SJ, Ashburner JM, Chang Y, Lester WT, Barry M, Grant RW. Population-based breast cancer screening in a primary care network. Am J Managed Care. 2012;18(12):821.

54. Ball K, Mouchacca J, Jackson M. The feasibility and appeal of mobile 'apps' for supporting healthy food purchasing and consumption among socioeconomically disadvantaged women: a pilot study. Health Promotion J Aust. 2014;25(2):79-82.

55. Choi J, hyeon Lee J, Vittinghoff E, \& Fukuoka Y. mHealth physical activity intervention: A randomized pilot study in physically inactive pregnant women. Maternal and Child Health Journal. 2016;20:5:1091-1101.

56. Fjeldsoe BS, Miller YD, Marshall AL. Social cognitive mediators of the effect of the MobileMums intervention on physical activity. Health Psychol. 2013;32(7):729.

57. Akinfaderin-Agarau F, Chirtau M, Ekponimo S, Power S. Opportunities and limitations for using new media and mobile phones to expand access to sexual and reproductive health information and services for adolescent girls and young women in six Nigerian states. Afr J Reprod Health. 2012;16(2):219-30.

58. Atkinson KM, Westeinde J, Ducharme R, Wilson SE, Deeks SL, Crowcroft N, Wilson K. Can mobile technologies improve on-time vaccination? A study piloting maternal use of ImmunizeCA, a Pan-Canadian immunization app. Human Vac Immunotherap. 2016;12(10):2654-61.

59. Brinkel J, May J, Krumkamp R, Lamshöft M, Kreuels B, Owusu-Dabo E, ... \& Fobil JN. Mobile phone-based interactive voice response as a tool for improving access to healthcare in remote areas in Ghana: an evaluation of user experiences. Trop Med Int Health 2017;22:5:622-630.

60. Fiander A, Ndahani C, Mmuya K, Vanneste T. Results from 2011 for the transportMYpatient program for overcoming transport costs among women seeking treatment for obstetric fistula in Tanzania. Int J Gynecol Obstetr. 2013;120(3):292-5.

61. Kukafka R, Yi H, Xiao T, Thomas P, Aguirre A, Smalletz C, ... \& Crew K. Why breast cancer risk by the numbers is not enough: Evaluation of a decision aid in multi-ethnic, low-numerate women. J Med Internet Res. 2015;17:7.

62. Potnis D. Culture's consequences: Economic barriers to owning mobile phones experienced by women in India. Telematics Inform. 2016;33(2):356-69.

63. Sjöström M, Umefjord G, Stenlund H, Carlbring P, Andersson G, Samuelsson E. Internet-based treatment of stress urinary incontinence: a randomised controlled study with focus on pelvic floor muscle training. BJU Int. 2013;112(3):362-72.

64. Song FW, West JE, Lundy L, Smith DN. Women, pregnancy and health information online: the making of informed patients and ideal mothers. Gender Soc. 2012;26(5):773-98.

65. Wen KY, Miller SM, Kilby L, Fleisher L, Belton TD, Roy G, Hernandez E. Preventing postpartum smoking relapse among inner city women: development of a theory-based and evidence-guided text messaging intervention. JMIR Res Protocols. 2014;3:2.

66. Frizzo-Barker J, Chow-White PA. "There's an App for That" Mediating mobile moms and connected careerists through smartphones and networked individualism. Feminist Media Stud. 2012;12(4):580-9.

67. Kim C, Draska M, Hess ML, Wilson EJ, Richardson CR. A web-based pedometer programme in women with a recent history of gestational diabetes. Diabet Med. 2012;29(2):278-83.

68. Parajuli R, Doneys P. Exploring the role of telemedicine in improving access to healthcare services by women and girls in rural Nepal. Telematics Inform. 2017;34(7):1166-76.

69. Bissonnette-Maheux V, Provencher V, Lapointe A, Dugrenier M, Dumas AA, Pluye P, Desroches $S$. Exploring women's beliefs and perceptions about healthy eating blogs: a qualitative study. J Med Internet Res. 2015;17:4.

70. Tyers AA Gender Digital Divide? Women learning english through ICTs in Bangladesh. In mLearn. 2012;94-100.

71. Albright CL, Steffen AD, Novotny R, Nigg CR, Wilkens LR, Saiki K, Brown WJ. Baseline results from Hawaii's Nā Mikimiki project: a physical activity 
intervention tailored to multiethnic postpartum women. Women Health. 2012;52(3):265-91.

72. Gilbert L, Shaw SA, Goddard-Eckrich D, Chang M, Rowe J, McCrimmon T, Epperson M. Project WINGS (Women Initiating New Goals of Safety): A randomised controlled trial of a screening, brief intervention and referral to treatment (SBIRT) service to identify and address intimate partner violence victimisation among substance-using women receiving community supervision. Criminal Behav Mental Health. 2015;25(4):314-29.

73. Min YH, Lee JW, Shin YW, Jo MW, Sohn G, Lee JH, ... \& Yu JH. Daily collection of self-reporting sleep disturbance data via a smartphone app in breast cancer patients receiving chemotherapy: A feasibility study. Journal of Medical Internet Research. 2014;16:5:e135.

74. Sriramatr S, Berry TR, Spence JC. An Internet-based intervention for promoting and maintaining physical activity: A randomized controlled trial. Am J Health Behav. 2014;38(3):430-9.

75. Steffen AM, Gant JR. A telehealth behavioral coaching intervention for neurocognitive disorder family carers. Int J Geriatr Psychiatry. 2016;31(2):195-203.

76. Takeuchi S, Horiuchi S. Randomised controlled trial using smartphone website vs leaflet to support antenatal perineal massage practice for pregnant women. Women and Birth. 2016;29(5):430-5.

77. Weinert C, Cudney S, Comstock B, Bansal A. Computer intervention: IIIness self-management/quality of life of rural women. Can J Nurs Res. 2014:46(1):26-43.

78. Ventura F, Sawatzky R, Öhlén J, Karlsson P, Koinberg I. Challenges of evaluating a computer-based educational programme for women diagnosed with early-stage breast cancer: a randomised controlled trial. Eur J Cancer Care. 2017;26:5.
79. Martinez-Brockman JL, Shebl FM, Harari N, Perez-Escamilla R. An assessment of the social cognitive predictors of exclusive breastfeeding behavior using the Health Action Process Approach. Soc Sci Med. 2017;182:106-16.

80. Kim HK, Niederdeppe J, Graham M, Olson C, Gay G. Effects of online self-regulation activities on physical activity among pregnant and early postpartum women. J Health Commun. 2015;20:101115-24.

81. Ehlers DK, Huberty JL, de Vreede GJ. Can an evidence-based book club intervention delivered via a tablet computer improve physical activity in middle-aged women? Telemed e-Health. 2015;21(2):125-31.

82. Dimond JP, Fiesler C, Bruckman AS. Domestic violence and information communication technologies. Interact Comput. 2011;23(5):413-21. https ://doi.org/10.1016/j.intcom.2011.04.006.

83. Henry N, Powell A. Technology-facilitated sexual violence: a literature review of empirical research. Trauma Violence Abuse. 2018;19(2):195-208. https://doi.org/10.1177/1524838016650189.

84. United Nations Statistics Division. Gender data: Sources, gaps, and measurement opportunities. 2017. http://www.data4sdgs.org/sites/default/ files/2017-09/Gender\%20Data\%20-\%20Data4SDGs\%20Toolbox\%20Mod ule.pdf

\section{Publisher's Note}

Springer Nature remains neutral with regard to jurisdictional claims in published maps and institutional affiliations.
Ready to submit your research? Choose BMC and benefit from:

- fast, convenient online submission

- thorough peer review by experienced researchers in your field

- rapid publication on acceptance

- support for research data, including large and complex data types

- gold Open Access which fosters wider collaboration and increased citations

- maximum visibility for your research: over 100M website views per year

At BMC, research is always in progress.

Learn more biomedcentral.com/submissions 